\title{
Discussion of the relationship between the aerosol extinction coefficient error and background noise
}

\author{
Nianwen Cao $\cdot$ Jianzhong Shi $\cdot$ \\ Fengkai Yang $\cdot$ Peng Yan
}

Received: 24 March 2012/Revised: 30 July 2012/Published online: 28 September 2012

(C) The Author(s) 2012. This article is published with open access at Springerlink.com

\begin{abstract}
This paper discusses the relationships among the aerosol extinction coefficient error (AECE), background noise, and distance associated with lidar measurements. The AECE calculation is explained in detail, revealing that the AECE is the product of background noise, range squared, and a relation function. The result of an AECE calculation that uses lidar measurements obtained in Nanjing, China, agrees with a calculation that uses a simulated lidar signal. The AECE equation is verified with lidar measurement data and a simulated lidar signal, indicating the AECE equation is reasonable.
\end{abstract}

\section{Introduction}

Aerosols are a source of uncertainty and an important cause of climate change. Aerosols also strongly pollute the atmospheric environment and are harmful to public health [1-3]. Consequently, scientists continue to consider aerosol research an important topic, as different aerosol optical properties cause different atmospheric radiation effects, and a database of aerosol optical properties has yet to be established in atmospheric research. High-accuracy aerosol optical properties are necessary for atmospheric studies. For instance, the aerosol extinction coefficient, and the

N. Cao $(\bowtie) \cdot$ J. Shi $\cdot$ F. Yang

School of Atmospheric Physics,

Nanjing University of Information Science and Technology,

Nanjing 210044, China

e-mail: nwcao@yahoo.com.cn

P. Yan

Center for Atmosphere Watch and Services,

CMA, Beijing 100081, China related visibility and optical depth, are essential in meteorology research [4]. Lidar can measure aerosols with high efficiency and range resolution. However, deficiencies still exist in lidar aerosol measurements. Aerosol measurement uncertainty exists for two reasons: the inversion method for the aerosol parameter, which has many assumptions [5], and systematic error due to the lidar system. Systematic error due to background noise is especially important, because it persists in aerosol lidar measurements. It remains an open question as to how background noise should be handled. Researchers commonly reduce background noise, experimentally, by making lidar measurements at night, using a narrow bandpass filter, and by decreasing the telescope's field of view. However, few studies have examined aerosol measurement error due to background noise [6]. This paper presents the theoretical calculation of the aerosol extinction coefficient error (AECE) due to background noise. The equation relating the AECE and background noise is calculated in detail. The AECE is calculated using experimental data and a simulated lidar signal.

\section{Theory of the AECE}

The aerosol extinction coefficient inversion equation is as follows [7]:

$\sigma(r, x)=\frac{\exp \left[\left(S(r, x)-S_{m}\right) / k\right]}{\sigma_{m}^{-1}+\frac{2}{k} \int_{r}^{r_{m}}\left\{\exp \left[\left(S(r, x)-S_{m}\right) / k\right]\right\} \mathrm{d} r}$

where $x$, a variable related to noise $\mathrm{d} p$, is introduced for ease of discussion $\left(\mathrm{d} p=\frac{\partial p}{\partial x} \mathrm{~d} x\right), r$ is the distance ( $x$ and $r$ are independent), $\sigma(r, x)$ is the aerosol extinction coefficient, $S(r, x)=\ln \left[r^{2} p(r, x)\right], p(r, x)$ is the lidar return signal, $\sigma_{m}$ is 

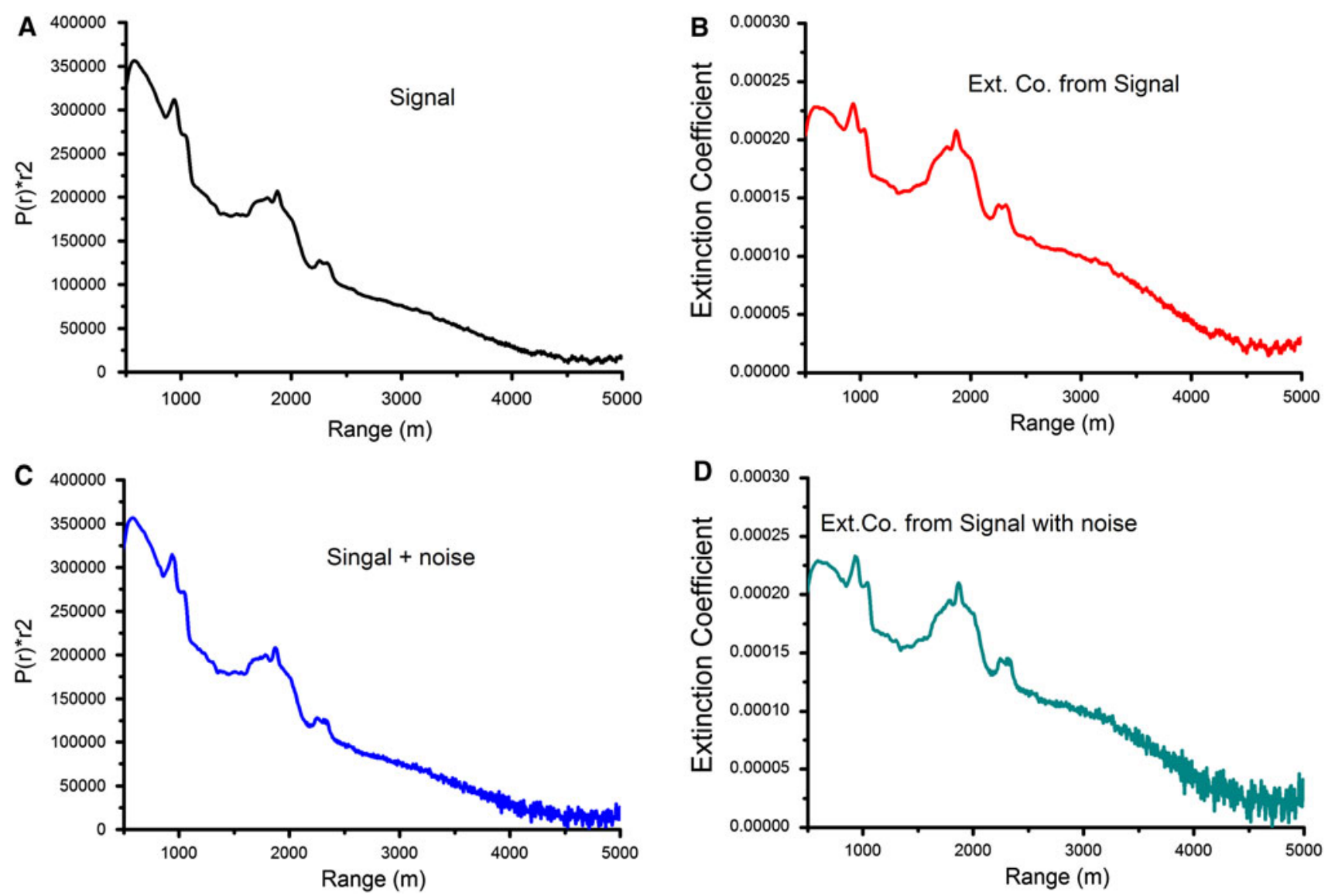

Fig. 1 Aerosol lidar return signal and inverse extinction coefficient

the boundary value of the extinction coefficient, $S_{m}$ is the boundary value of $S$, and $k$ is a constant. Therefore,

$$
\begin{aligned}
& {\left[S(r, x)-S\left(r_{m}, x_{m}\right)\right] / k=\left\{\ln \left[r^{2} p(r, x)\right]-\ln \left[r_{m}^{2} p\left(r_{m}, x_{m}\right)\right]\right\} / k} \\
& =\ln \left[\frac{r^{2} p(r, x)}{r_{m}^{2} p\left(r_{m}, x_{m}\right)}\right]^{\frac{1}{k}}
\end{aligned}
$$

and

$\exp \left[\left(S(r, x)-S\left(r_{m}, x_{m}\right)\right) / k\right]=\left[\frac{r^{2} p(r, x)}{r_{m}^{2} p\left(r_{m}, x_{m}\right)}\right]^{\frac{1}{k}}$.

Using (2) and (3) in (1), we obtain

$\sigma(r, x)=\frac{\left[\frac{r^{2} p(r, x)}{r_{m}^{2} p\left(r_{m}, x_{m}\right)}\right]^{\frac{1}{k}}}{\sigma_{m}^{-1}+\frac{2}{k} \int_{r}^{r_{m}}\left[\frac{r^{2} p(r, x)}{r_{m}^{2} p\left(r_{m}, x_{m}\right)}\right]^{\frac{1}{k}} \mathrm{~d} r}$.

For convenience, the functions $U(x)$ and $V(x)$ are introduced as follows:

$U(x)=\left[\frac{r^{2} p(r, x)}{r_{m}^{2} p\left(r_{m}, x_{m}\right)}\right]^{\frac{1}{k}}$
$V(x)=\sigma_{m}^{-1}+\frac{2}{k} \int_{r}^{r_{m}}\left[\frac{r^{2} p(r, x)}{r_{m}^{2} p\left(r_{m}, x_{m}\right)}\right]^{\frac{1}{k}} \mathrm{~d} r$.

Taking the differentials of (5) and (6) with respect to $x$ we obtain

$\mathrm{d} U(x)=\frac{1}{k}\left[\frac{r^{2} p(r, x)}{r_{m}^{2} p\left(r_{m}, x_{m}\right)}\right]^{\frac{1}{k}-1} \frac{r^{2} \mathrm{~d} p}{r_{m}^{2} p\left(r_{m}, x_{m}\right)}$

and

$\mathrm{d} V(x)=\frac{2}{k} \frac{1}{k} \int_{r}^{r_{m}}\left[\frac{r^{2} p(r, x)}{r_{m}^{2} p\left(r_{m}, x_{m}\right)}\right]^{\frac{1}{k}-1} \frac{r^{2} \mathrm{~d} p}{r_{m}^{2} p\left(r_{m}, x_{m}\right)} \mathrm{d} r$.

Using (5)-(8), the differential of (4) can be written as

$d \sigma(x)=\frac{d U(x) V(x)-U(x) d V(x)}{V^{2}(x)}$

Setting $k=1$ in (9), we obtain

$\mathrm{d} \sigma(x)=T(r, x) r^{2} \mathrm{~d} p$

where 


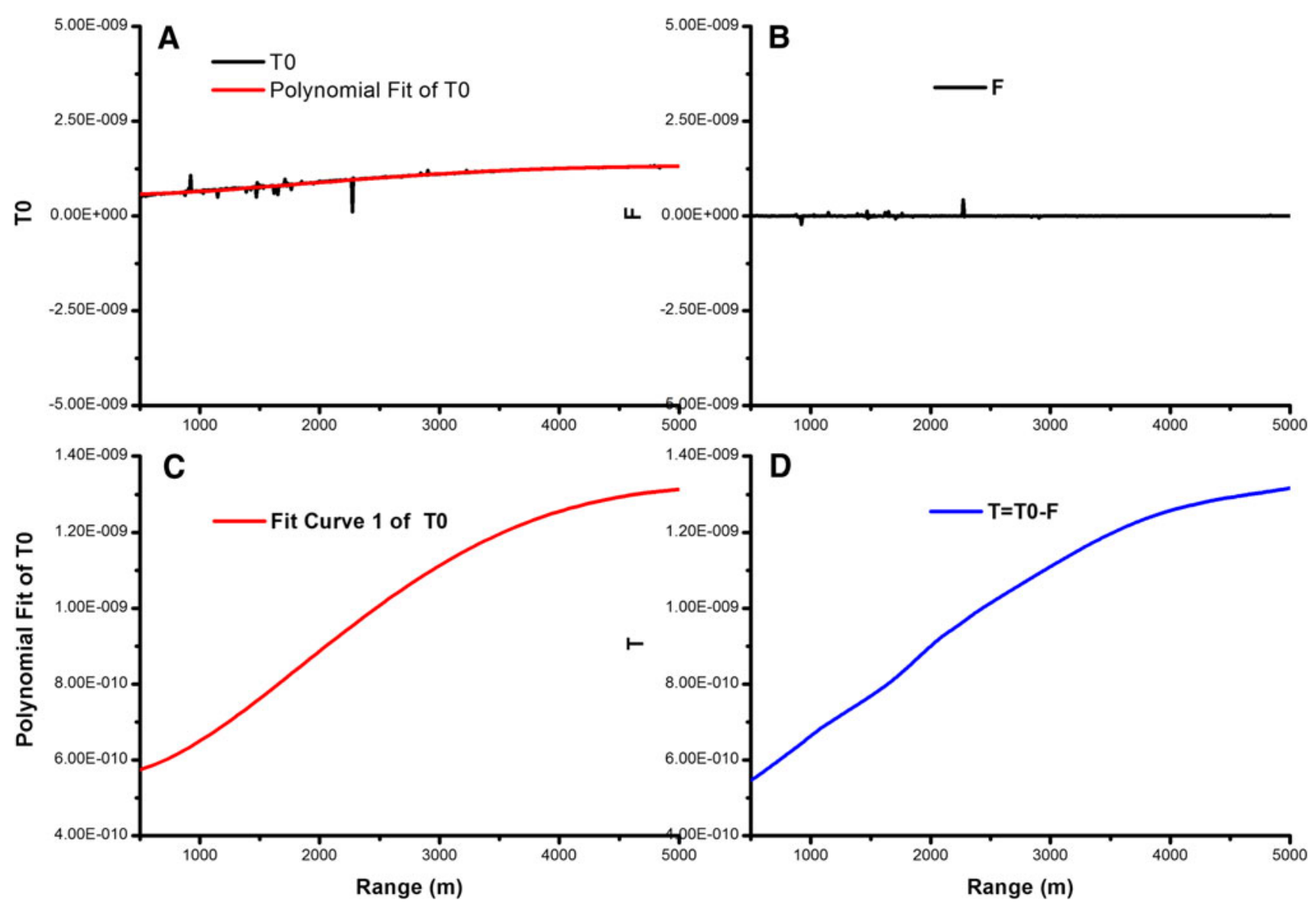

Fig. 2 a-d The function $T_{0}(r, x)$ calculated from experimental data and a polynomial fit, the function $F(r, x)$, the polynomial fit of $T_{0}(r, x)$, and the relation function $T(r, x)$

$T(r, x)=T_{0}(r, x)-F(r, x)$

where

$$
\begin{aligned}
& T_{0}(r, x)=\frac{1}{\sigma_{m}^{-1} r_{m}^{2} p\left(r_{m}, x_{m}\right)+2 \int_{r}^{r_{m}} r^{2} p(r, x) d r} \\
& F(r, x)=\frac{\frac{2 p(r, x)}{\mathrm{d} p} \int_{r}^{r_{m}} r^{2} \mathrm{~d} p \mathrm{~d} r}{\left[\sigma_{m}^{-1} r_{m}^{2} p\left(r_{m}, x_{m}\right)+2 \int_{r}^{r_{m}} r^{2} p(r, x) \mathrm{d} r\right]^{2}}
\end{aligned}
$$

We discuss the relationships among $T(r, x), T_{0}(r, x)$, $F(r, x)$ with reference to experimental and simulation data, as described below. The calculation results show that $F(r, x)$ is negligibly small.

\section{Analysis of aerosol lidar measurement data}

Aerosol lidar measurement data and related analysis are shown in Fig. 1. In lidar measurements, the background noise is obtained using a cover over the top of the telescope to block scattered light. We handle the background noise according to (14), and compute $\overline{p(r, x) \times r^{2}}=\frac{\sum_{i=1}^{n}\left(p_{i}(r, x)-\text { noise }\right)}{n} \times r^{2}$

According to (14), we subtract the background noise from the experimental data, and then apply smoothing to obtain the range-corrected signal (the lidar signal multiplied by range squared) without noise (Fig. 1a). We subtract the background noise from the experimental data to obtain a lidar signal without noise. Figure $1 \mathrm{~b}$ shows the extinction coefficient obtained via inversion from the signal in Fig. 1a. Because the return lidar signal without background noise is smooth, the corresponding AECE profile in Fig. 1b is also smooth. Figure 1c shows the aerosol lidar range-corrected signal with background noise. Figure $1 \mathrm{~d}$ is the extinction coefficient profile obtained via inversion from the noisy lidar signal in Fig. 1c. The extinction coefficient profile in Fig. 1d has more fluctuation with increasing range than does that in Fig. 1b. This result indicates that the aerosol extinction coefficient fluctuation (extinction coefficient error) is mainly due to background noise. According to the above theory, (10) is the relationship between AECE and background noise, range, and the relation function $T(r, x)$. Equations (11)-(13) 


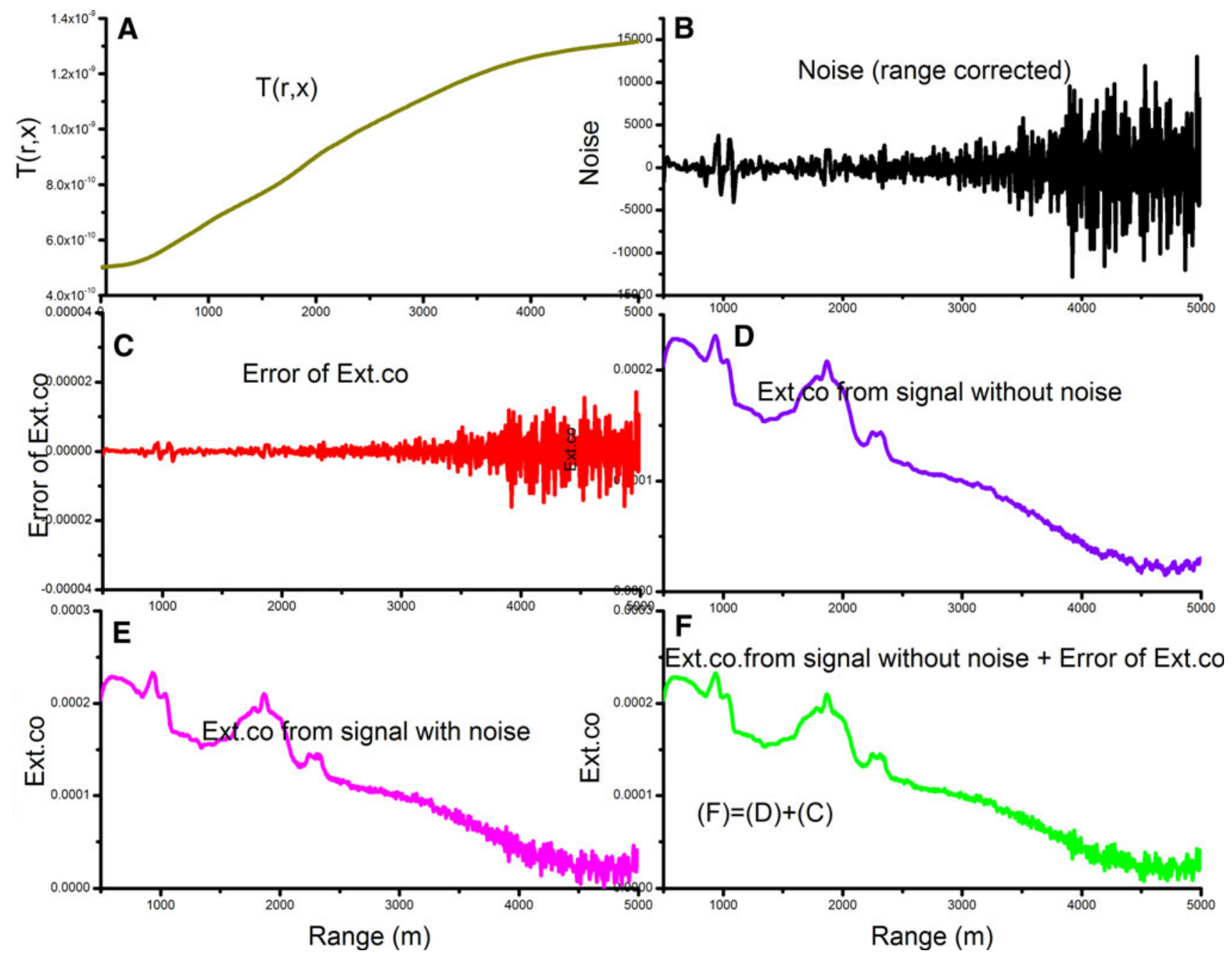

Fig. 3 a Relation function $T(r, x)$. b Range-corrected noise signal (noise signal intensity multiplied by range squared). c Aerosol extinction coefficient error (AECE) calculated from (10). d Extinction
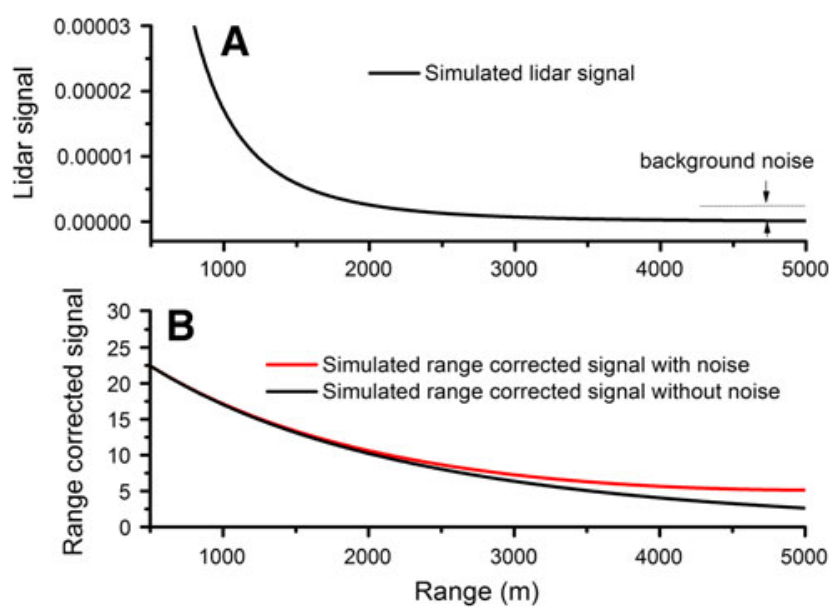

Fig. 4 a Simulated return lidar signal. b Simulated range-corrected signal with and without background noise. The background noise is the assumed constant coefficient calculated from the signal without noise. e Extinction coefficient calculated from the signal with noise. $\mathbf{f}$ Sum of $\mathbf{d}$ and $\mathbf{c}$
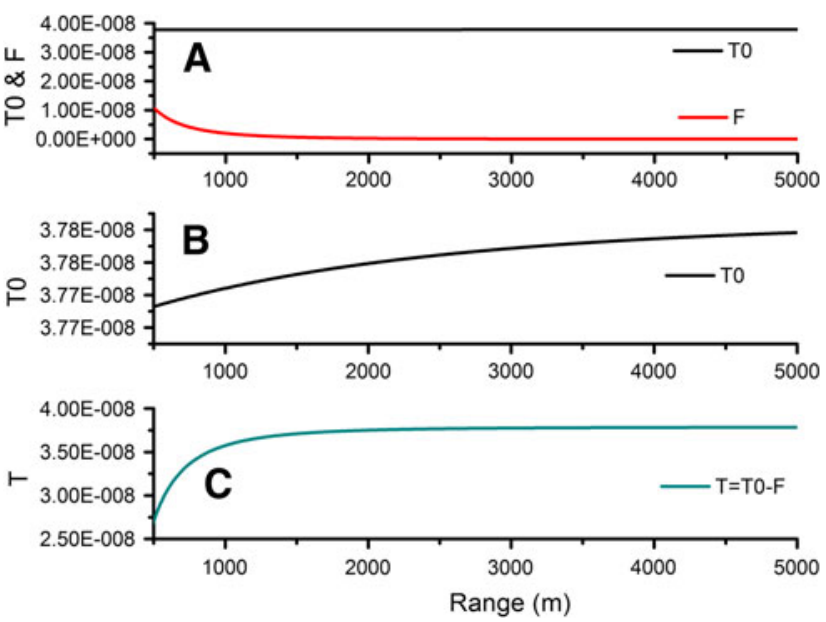

Fig. 5 a Functions $T_{0}(r, x)$ and $F(r, x)$ calculated using simulation data (see Fig. 4). b Enlargement of a, showing the function $T_{0}(r, x)$. c Function $T(r, x)$ calculated using simulation data 


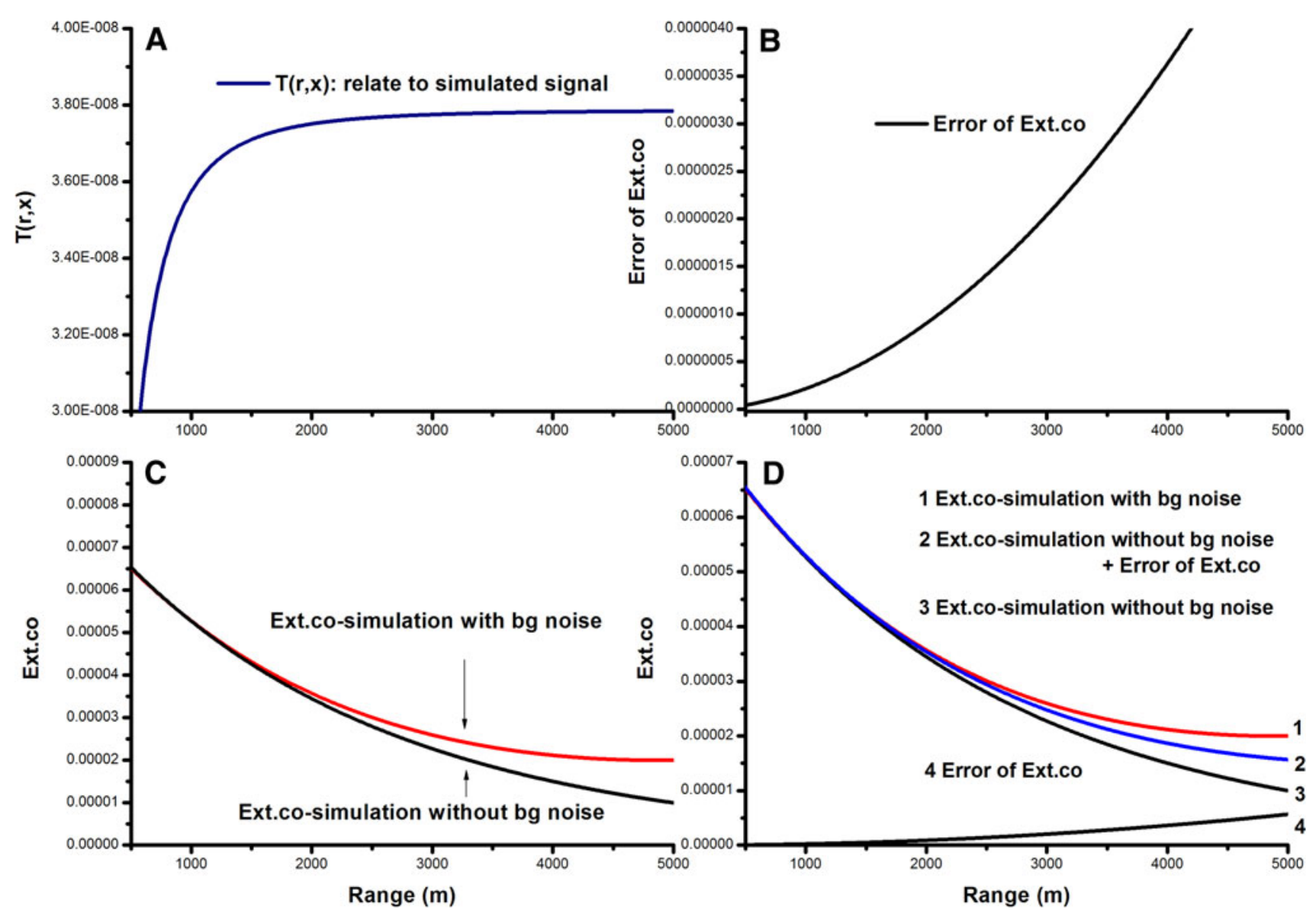

Fig. 6 Extinction coefficient calculated from the simulated signal. a Relation function $T(r, x)$. b Error of the extinction coefficient calculated from (10) using the simulated signal. c Extinction

indicate that $T(r, x)$ depends on the lidar return signal and range. Using measurement data, $T(r, x)$ is calculated and shown in Fig. 2. According to (12), the function $T_{0}(r, x)$ is calculated using experimental data $p(r, x)$ and $p\left(r_{m}, x_{m}\right)$, small fluctuations in $T_{0}(r, x)$ are due to noise in the experimental data, and we performed a polynomial fit calculation for $T_{0}(r, x)$, as shown in Fig. 2a. According to (13), the function $F(r, x)$ is calculated using experimental data, small fluctuations in $F(r, x)$ are due to noise in the experimental data, and the value of $F(r, x)$ is close to zero, as shown in Fig. 2b. Figure $2 c$ is the same as Fig. 2a but with the vertical axis enlarged, showing the polynomial fit calculation for $T_{0}(r, x)$. According to (11), the relation function $T(r, x)$ differs between $T_{0}(r, x)$ and $F(r, x)$, as shown in Fig. 2 d.

It is clear that $T(r, x)$ is almost equal to $T_{0}(r, x)$ and that $F(r, x)$ is negligibly small. Figure 3 shows the relationships among AECE, $T(r, x)$, and background noise. Figure $3 \mathrm{a}$ shows that $T(r, x)$ increases with increasing range; consequently, AECE also increases in this way. The background noise signal was measured immediately coefficient profiles corresponding to the simulated signal with and without background signal. d Profiles 1 and 3 are those in c; profile 4 is the error shown in $\mathbf{b}$; profile 2 shows the sum of profiles 3 and 4

after each aerosol measurement, and is shown in Fig. $3 b$. The AECE, calculated according to (10), is proportional to the background noise and becomes larger with increasing range (Fig. 3c). We subtracted background noise from measurement data to obtain an aerosol measurement signal without background noise. Figure $3 d$ shows the extinction coefficient calculated from the aerosol measurement signal without background noise, and Fig. 3e shows the extinction coefficient calculated from the lidar signal with background noise, using the following equation:

$\sigma($ lidarsignal + noise $)=\sigma($ lidarsignal $)+\mathrm{d} \sigma$

where $\sigma$ (lidarsignal + noise $)$ and ( $\sigma$ (lidarsignal $))$ are the aerosol extinction coefficients calculated from the lidar signal with and without background noise, respectively, and $\mathrm{d} \sigma$ is the AECE. Using (15), and thus adding the data from Fig. 3c, d, we obtain the aerosol extinction coefficient profile (Fig. 3f). The similarity of the profiles in Fig. $3 \mathrm{f}$ and $3 \mathrm{e}$ indicates the validity of equation (10), from which the result shown in Fig. $3 \mathrm{c}$ was obtained. 


\section{Simulations}

To verify the AECE theory further, we performed additional simulations. Figure $4 \mathrm{a}$ shows the simulated lidar return signal without background noise, and the background itself, which is assumed to be the constant, while Fig. $4 \mathrm{~b}$ shows the range-corrected signal with and without background noise. Based on simulations, $T(r, x), T_{0}(r, x)$, and $F(r, x)$ are calculated as in Fig. 5 .

Figure 5a shows $T_{0}(r, x)$ and $F(r, x)$. Figure $5 \mathrm{~b}$ is the same as Fig. 5a but with the vertical axis expanded, showing $T_{0}(r, x)$. Figure 5c shows $T(r, x)$-the difference between $T_{0}(r, x)$ and $F(r, x) . F(r, x)$ is negligibly small (Fig. 5a) and $T(r, x)$ is almost equal to $T_{0}(r, x)$, similar to Fig. 2b. Using the simulation data in Fig. 4 and $T(r, x)$ in Fig. 5, AECE can be calculated as in Fig. 6. Figure 6a shows that the relation function $T(r, x)$ is similar to that in Fig. 3a, as they both increase with increasing range. $T(r, x)$ in both Figs. 6a and $3 \mathrm{a}$ is calculated using (11); however, $T(r, x)$ in Fig. 3a (Fig. 6a) is based on the measurement (simulated) data. Employing (10), we use the simulated data to calculate the extinction coefficient error (AECE) due to background noise (Fig. 6b). The extinction coefficient error increases with distance, assuming the background noise is constant (Fig. 4a). The extinction coefficient error shown in Fig. $6 \mathrm{~b}$ corresponds to that in Fig. 3c. The AECE shown in Fig. 3c relates to the experimental noise. The two extinction coefficient profiles shown in Fig. 6c are based on the simulated data, and correspond to the range-corrected signals with and without background noise, which are shown in Fig. $4 \mathrm{~b}$.

The extinction coefficient profile 1 (profile 3) in Fig. 6d is calculated from the simulated signal with constant (without) background noise. Profile 4 in Fig. 6d is the error of the extinction coefficient. The extinction coefficient, profile 2 in Fig. 6d, is the sum of profiles 3 and 4. Recall that profile 1 is the extinction coefficient calculated using (1), and profile 2 is the extinction coefficient calculated from the full AECE theory. Figure 6 shows a small difference between profiles 1 and 2 .

\section{Conclusions}

In aerosol lidar measurements, the measurement accuracy is of great importance. The accuracy of aerosol optical property depends on several factors, including the boundary value and parameter assumptions, and background noise. According to lidar theory, the relationship between the AECE and background noise, and distance can be calculated. The AECE is proportional to the square of distance $r^{2}$, the relation function $T(r, x)$, and the amount of background noise. Here, the relationship between the AECE and background noise has been verified by using aerosol lidar measurements and simulated lidar signals.

Acknowledgments This work was supported by the Nature Science Foundation of China under Project 41175033/D0503 and Chinese Public Welfare Industry Special Project GYHY 201006047-5.

Open Access This article is distributed under the terms of the Creative Commons Attribution License which permits any use, distribution, and reproduction in any medium, provided the original author(s) and the source are credited.

\section{References}

1. R.K. Pachauri, A. Reisinger, Climate Change (IPCC, Geneva, 2007), p. 104

2. N. Cao, S. Li, T. Fukuchi, T. Fujii, R.L. Collins, Z. Wang, Z. Chen, Appl. Phys. B 85, 163-167 (2006)

3. N. Cao, T. Fuckuchi, T. Fujii, R.L. Collins, S. Li, Z. Wang, Z. Chen, Appl. Phys. B 82, 141-148 (2006)

4. W. Viezee, E.E. Uthe, R.T. Collis, J. Appl. Meteorol. 8, 274 (1969)

5. R.T.H. Collis, Q.J.R. Meteorol. Soc. 92, 220 (1966)

6. N. Cao, T. Fuckuchi, T. Fujii, Z. Chen, J. Huang. J. Electromagn, Anal. Appl. 2(7), 450-456 (2010)

7. J.D. Klett, Appl. Opt. 20(2), 211-219 (1981) 